\title{
Faccionalismo e integración vertical en contextos multinivel. El caso del Partido Nacionalista Vasco*
}

\author{
Raúl Gómez \\ Santiago Pérez-Nievas \\ Instituto Universitario de Florencia \\ Instituto Juan March de Estudios e Investigaciones \\ rgomez@march.es
}

\section{Resumen}

Los determinantes de los niveles de integración vertical en los partidos políticos y su influencia sobre las estrategias de las facciones internas de los partidos han sido poco estudiados en la literatura, y los pocos trabajos que lo han hecho se han centrado en partidos de ámbito estatal. Aquí argumentamos que la organización de los partidos afecta de forma definitiva al comportamiento de las facciones internas, de tal forma que el faccionalismo tendrá un carácter más territorializado en partidos con bajos niveles de integración. Para ilustrar esta hipótesis, empleamos la evidencia empírica de un partido de ámbito no estatal (PANE): el Partido Nacionalista Vasco.

Palabras clave: partido de ámbito no estatal, faccionalismo, integración vertical, descentralización.

\section{Abstract. Factionalism and vertical integration in multilevel contexts. The case of the Basque Nationalist Party}

The determinants of the distinct levels of party vertical integration and their influence on internal factions' strategies have been certainly understudied so far, and those few works attempting to do it have focused on statewide political parties. Here we claim that party organization eventually affects the behavior of internal factions, so that factionalism will have a more territorial character in parties with low levels of vertical integration than in the rest. This argument is then illustrated with empirical evidence from one non-statewide party: the Basque Nationalist Party,

Key words: non-statewide political party, factionalism, vertical integration, decentralization.

Agradecemos a la Comunidad de Madrid y a la Universidad Autónoma de Madrid (UAM) la financiación que, en el marco del proyecto sobre Participación politica y electoral en sistemas multinivel (CCG07-UAM/HUM-1598), ha hecho posible esta investigación. 


\section{Sumario}

Introducción

Faccionalismo e integración vertical

Hipótesis

La integración vertical en distintos PANE del sistema político español

La organización del PNV desde 1977 hasta 1987
La organización territorial del faccionalismo en el PNV

Las consecuencias de la ruptura sobre el grado de integración vertical del partido

Conclusiones

Referencias bibliográficas

\section{Introducción}

El Euzko Alderdi Jeltzalea (Partido Nacionalista Vasco, en adelante, PNV) es un partido político de ámbito no estatal (PANE) que no sólo ostenta representación a nivel local, provincial y autonómico, tanto en el País Vasco como en Navarra, sino que, además, ha presidido de forma ininterrumpida el Gobierno Vasco desde las primeras elecciones autonómicas. Se trata, además, de un actor político cuyo papel en la arena estatal ha resultado fundamental, al haber sido, junto con otros PANE, socio parlamentario de varios gobiernos españoles (legislaturas de 1993 y 1996, hasta el momento). Todo ello hace del PNV un partido importante sobre cuyo funcionamiento, al igual que ocurre con otros PANE, se conoce realmente poco.

Aparte de lo anterior, existen varias características que hacen a este partido interesante para poner a prueba diversas hipótesis. El PNV no sólo es una de las organizaciones partidistas cuyas unidades territoriales tienen mayor grado de autonomía en nuestro sistema político, sino que cuenta, además, con altos niveles de faccionalismo, sobre todo hasta 1986, año en que se produce la escisión que da lugar al surgimiento de EA. Estas características resultan útiles para poder poner a prueba varias hipótesis concernientes a las dinámicas del faccionalismo en partidos políticos según sus características organizativas.

Si bien investigaciones anteriores sobre el PNV han puesto de relieve algunos de los aspectos a analizar aquí (De Pablo y Mees, 2005; Pérez-Nievas, 2002), este trabajo pretende, por un lado, aportar nuevas hipótesis y mecanismos causales generalizables a otros partidos políticos, y, por otro lado, realizar un análisis más exhaustivo de la regulación estatutaria concerniente a las distintas dimensiones de la integración vertical dentro del mismo.

El trabajo está estructurado de la siguiente manera. En la próxima sección, introducimos la literatura que se ha dedicado a la integración vertical, así como aquélla que ha prestado atención a las relaciones entre ésta y el faccionalismo. Tras ello, expondremos brevemente las hipótesis planteadas y los mecanismos que subyacen a las mismas. A continuación, explicamos las razones que nos han llevado al estudio de este caso en concreto y comparamos el grado de integración vertical de varios PANE. En el apartado siguiente, detallamos la regulación de la integración vertical que establecen los estatutos del PNV en el 
periodo 1977-1987, para pasar, con posterioridad, al análisis del desarrollo del faccionalismo en dicha época. Tras esto, analizamos los cambios estatutarios adoptados por el PNV en 1987 en relación con la situación anterior. Finalmente, en el último apartado, esbozaremos algunas conclusiones del análisis junto con los pasos que deben darse para el desarrollo de futuras investigaciones sobre el tema.

\section{Faccionalismo e integración vertical}

Al contrario de lo que ocurre con el faccionalismo, el estudio de la organización de los partidos a nivel territorial y la interrelación existente entre los distintos niveles es relativamente nuevo. Los principales autores que han prestado atención al fenómeno nos ofrecen multitud de clasificaciones de los distintos tipos de partido en función de las relaciones que se establecen entre sus estructuras centrales y territoriales (Carty, 1988; Dyck, 1991; Smiley, 1987). La mayoría de ellos, sin embargo, parten de la adopción de la expresión «integración vertical», acuñado por Smiley (1987), quien consideraba que un modelo «integrado» de partido es aquél en que los territorios actúan como correas de transmisión de los órganos centrales. Thorlakson (2001), sin embargo, propone que el grado de integración vertical también puede ser considerado como un continuum que va desde un grado ínfimo de relaciones entre los distintos niveles - lo que se correspondería con un modelo de partido truncado-, hasta un grado tal en el que prácticamente es el nivel central del partido el que toma todas las decisiones relevantes - modelo unitario-. En este trabajo, consideraremos también la integración vertical como un continuum, definiéndola como el grado de reparto de funciones y competencias entre los distintos niveles territoriales en que se organizan los partidos. De este modo, entenderemos que, a mayor grado de integración vertical, mayor será la proporción de dichas funciones y competencias que están en manos de los órganos centrales del partido, en detrimento de los órganos territoriales del mismo. Y viceversa, cuanta mayor autonomía tengan éstos últimos, menor será el grado de integración vertical.

En la última década, han proliferado varias investigaciones que tratan de explicar las causas de la variación encontrada en el grado de integración vertical, a menudo relacionándolo con el grado de descentralización del sistema político (Barnea y Rahat, 2007; Deschouwer, 2003; Hopkin, 2003; Hopkin y Van Biezen, 2006; Hough y Jeffery, 2006; Laffin, Shaw y Taylor, 2007), y son también varios los trabajos que, de entre los anteriores, se centran en partidos del sistema político español (Astudillo, en prensa; Betanzo, 2006; Méndez, 2004; Méndez y Orte, en prensa; Pérez-Nievas y Ramiro-Fernández, en prensa). Es cierto, sin embargo, que escasean los trabajos que consideren partidos de ámbito no estatal, si bien investigaciones más recientes han incorporado también a este tipo de partidos en el análisis, ya sea en exclusiva (Barberà, Sallas y Terrades, 2007), ya sea conjuntamente con partidos de ámbito estatal (Gómez, 2007). 
El interés por la integración vertical ha sido, sin embargo, relativamente tardío. Por ello, no sorprende que las relaciones entre aquélla y el faccionalismo se encuentren, en gran medida, poco estudiadas. Algunos trabajos pioneros a este respecto son Ştefuriuc y Verge (2008) y Verge (2007), que analizan el caso de Izquierda Unida (IU) argumentando que la facción dominante del partido hace uso del nivel de autonomía de las subunidades territoriales para llevar a cabo, en la arena autonómica, las estrategias que, a nivel central, no puede desarrollar, por encontrarse bloqueadas por las facciones minoritarias, que representan prácticamente la mitad de los miembros de los órganos federales. La lucha entre las diferentes facciones parece librarse, por tanto, en gran medida, en términos territoriales y, en su opinión, está relacionada con algunos de los cambios que se producen en los niveles de integración vertical del partido.

\section{Hipótesis}

Existen fundamentalmente dos fuentes distintas de recursos que pueden incrementar la importancia de los territorios dentro de los partidos y, por lo tanto, el interés de las élites por controlarlos. La primera de dichas fuentes es externa, y está relacionada con el grado de descentralización del Estado. A mayor nivel de descentralización, mayor es la importancia de las arenas políticas locales, lo que implica mayores oportunidades para designar cargos públicos en dichos ámbitos, gestionar distintos niveles de gobierno o tener acceso a financiación y a medios de comunicación propios de cada territorio.

Pero existe otra fuente de recursos que es interna y que se deriva de la estructura organizativa del partido. Cuando se proporciona un mayor nivel de autonomía a las subunidades territoriales, con ello se crean también nuevas arenas de competición intrapartido. El esquema clásico de partido político de masas donde los líderes hacen carrera dentro de los distintos niveles perfectamente jerarquizados hasta llegar al nivel central, probablemente quiebre en partidos menos integrados verticalmente, dado que las organizaciones territoriales cuentan con un mayor número de recursos y adquieren, por tanto, mayor relevancia (Hopkin y Van Biezen, 2006).

Si las fuentes externas e internas están relacionadas o no, no es objeto de estudio en este trabajo en concreto. Aunque reconocemos que dicha relación existe, consideraremos la organización del partido como dada, lo que nos permitirá centrar nuestro interés únicamente en la influencia directa que las fuentes internas pueden tener sobre las estrategias desplegadas por las élites del mismo y, en concreto, por las distintas facciones. Nuestro argumento es que la dinámica de las relaciones entre ellas no se puede entender sin tener en cuenta la forma de organizarse del propio partido, que condiciona, como institución, los movimientos de los actores que forman parte del mismo.

Para analizar los incentivos de las diferentes facciones dentro de un partido, nos detendremos primero en aquéllas que no conforman la facción dominante central, y luego en las que sí. 
Para las facciones minoritarias, que no forman parte de la facción dominante del partido, la autonomía de los territorios es importante a la hora de desarrollar sus distintas estrategias. En un partido con alta integración vertical, el centro tiene una gran capacidad de imponer decisiones y, por tanto, cualquier estrategia basada en concentrarse territorialmente sería muy arriesgada para las facciones minoritarias, dado que la intervención central podría hacerlas saltar por los aires sin demasiado esfuerzo. Es probable, por lo tanto, que, en este tipo de partidos, estas facciones opten por concentrarse en la lucha por el poder a nivel central, expandiéndose de forma transversal a través de los territorios. Sin embargo, si éstos últimos cuentan con amplia autonomía, controlar una organización territorial puede resultar tremendamente útil para una facción. Poniendo un mayor esfuerzo en controlar territorios concretos, las facciones minoritarias pueden hacerse fácilmente con recursos organizativos, políticos y económicos que les garanticen la supervivencia y les permitan afrontar con mayor seguridad la batalla por el poder a nivel central.

Pasemos ahora a la facción dominante central. En partidos con altos niveles de integración vertical, el centro podrá intervenir con facilidad sobre cualquier territorio atacando a las facciones díscolas que se puedan concentrar en ellos. Sin embargo, ocurrirá lo contrario en partidos donde los territorios tengan mayor autonomía. En dicho contexto, el poder de los órganos centrales es limitado y, por lo tanto, el conflicto se trasladará a los territorios, donde las facciones intentarán mantener a toda costa su hegemonía. En consecuencia, la primera hipótesis será la siguiente:

$\mathrm{H} 1$ : en partidos políticos con bajos niveles de integración vertical, el faccionalismo tenderá a territorializarse.

Ahora bien, ¿no responderá entonces la facción dominante cambiando las reglas del juego y aumentando el nivel de integración vertical del partido? Si dicha facción fuera homogénea, ésta sería la situación más probable. Es posible, sin embargo, que varios de los territorios que la conforman tengan también incentivos para mantener o aumentar su nivel de autonomía. Así, la presencia de elementos idiosincráticos al territorio en la competición política —en otras palabras, la presencia de la dimensión centro-periferia- puede empujar a las organizaciones territoriales a reivindicar mayor capacidad para modular el discurso y la acción del partido, a fin de maximizar sus resultados electorales en la arena local. Estos territorios deberán, entonces, optar entre aumentar el poder del centro para atacar a las facciones díscolas, con lo cual disminuirá su propia autonomía, o incrementar la autonomía de los territorios, con lo cual darán, entonces, más recursos también a aquéllos que se encuentran controlados por facciones minoritarias del partido.

Contamos, por tanto, con tres posibles tipos de actores: las facciones minoritarias, que preferirán altos niveles de autonomía, y los territorios controlados por la facción dominante, algunos de los cuales tendrán incentivos para restringir el grado de integración vertical, mientras que otros los tendrán para aumentarlo. Por ello, la existencia de alianzas transversales a este respecto 
entre territorios de facciones distintas para mantener el statu quo es más que probable. Es posible, incluso, que la facción dominante acabe por preferir una suerte de integración vertical asimétrica de facto, de tal manera que las organizaciones afines a ella puedan desarrollar con autonomía aquellas políticas que, a nivel central, puedan estar bloqueadas en casos de niveles altos de faccionalismo, mientras se acudirá a todos los mecanismos posibles para mantener bajo control al resto de organizaciones. En todo caso, lo interesante es analizar qué ocurriría si el conflicto se saldara con la salida de una de las partes, porque ello nos daría cierta evidencia prima facie a favor o en contra de los mecanismos propuestos. De forma directa y concisa: si es cierto que las facciones minoritarias tienen incentivos para reclamar una mayor autonomía de los territorios del partido, entonces la salida de dichas facciones debería provocar un cambio brusco en la relación de fuerzas en favor de un mayor grado de integración vertical. Y esto no sólo porque la facción mayoritaria querrá prevenirse de que facciones ulteriores puedan emplear los mismos recursos, sino también porque las posibles alianzas transversales entre territorios afines a la facción dominante y el resto desaparecerían, lo cual sesgaría el equilibrio en dirección a una mayor integración vertical. Por tanto, en respuesta a un reciente conflicto que se salda de forma traumática, el nivel de integración vertical deberá aumentar.

$\mathrm{H} 2$ : Si el conflicto se resuelve de forma traumática y acaba con la salida de las facciones minoritarias, entonces, la correlación de fuerzas será más favorable a las tesis del centro y el nivel de autonomía de los territorios descenderá.

Para sostener este resultado, debemos partir de un supuesto no descabellado, y es que ninguna facción del partido que constituya la mayoría central querrá vaciar de contenido el poder del centro que ella misma ostenta. Incluso en el caso de que, tras la salida de las facciones minoritarias, la mayoría de la anterior facción dominante esté constituida por territorios que tienen incentivos electorales para descentralizar el poder, mantener niveles bajos de integración puede suponer facilitar el desarrollo y el fortalecimiento de facciones ulteriores. Y la percepción del riesgo de que esto ocurra probablemente será extremadamente alta tras una escisión reciente.

\section{La integración vertical en distintos PANE del sistema político español}

Independientemente del ámbito en que actúen, los partidos políticos cuentan con muy distintos grados de integración vertical. Mientras que unos de ellos dan mayor autonomía a las subunidades territoriales en que se conforman, otros concentran todo el poder en el centro, de manera que las unidades inferiores son meras correas de transmisión.

La mayor parte de la literatura coincide en señalar que, para estudiar el grado de integración vertical en un partido político, es necesario tener información al respecto de varios indicadores, que Méndez y Orte (en prensa) agru- 
pan en tres dimensiones: la organización interna del partido, la oferta política y las estrategias.

En cuanto a la primera dimensión, la organización interna, ésta hace referencia a la distribución del poder territorial versus el poder central dentro del partido, que puede ser medida a través de varios indicadores: la gestión de los recursos (organizativos y financieros), la regulación de sus normas, la resolución de conflictos y la composición de los órganos que toman la mayor parte de las decisiones del partido a nivel central.

La segunda dimensión mide el grado en que los territorios tienen autonomía para establecer la oferta política. Esta dimensión se compone de tres indicadores: la elaboración del programa; el tipo de mecanismos empleados para coordinar las políticas, y, por último, otras situaciones que refuercen la imagen autónoma de cada territorio (o de algunos) de cara al electorado. A este respecto, se considerará más integrado un partido que centraliza todas las decisiones y tiene una imagen homogénea pero que cuenta con algún territorio cuya autonomía es mayor, que otro en el que todos los territorios cuenten con amplio margen de autonomía.

La tercera dimensión mide el aspecto más estratégico de los partidos en su papel para seleccionar élites y conformar gobiernos, esto es, la autonomía de los territorios a la hora de establecer la composición de las candidaturas, así como de conformar coaliciones preelectorales o postelectorales ${ }^{1}$.

A fin de comparar niveles de integración vertical entre varios partidos, hemos elaborado un índice para cada una de las dimensiones anteriormente mencionadas, atendiendo a la información contenida en los estatutos de los mismos. Para ello, hemos otorgado a cada uno de los indicadores un valor entre 1 y 5 , donde el 5 significa que no existe autonomía de las unidades territoriales y el 1 que existe autonomía plena, tal y como muestra la tabla 1.

Tabla 1. Criterios de asignación de valores en una escala de integración vertical

1. Todo el peso recae sobre las unidades territoriales.

2. Los territorios tienen más peso que el centro

3. Centro y territorio tienen un peso equivalente.

4. El centro tiene más peso que los territorios.

5. Todo el peso recae sobre los órganos centrales.

1. En cuanto a la composición de las candidaturas, el hecho de situarla aquí difiere en relación con la clasificación de Méndez y Orte (en prensa) y puede ser objeto de discusión teórica. Dichos autores incluyen la selección de candidatos en la dimensión correspondiente a las políticas y a los programas de los partidos. Desde nuestro punto de vista, sin embargo, debemos distinguir — al menos teóricamente- entre los aspectos relacionados con el contenido político (la oferta electoral) y aquéllos que tienen que ver con el partido en las instituciones, como son el hecho de seleccionar representantes y de guiar sus relaciones con representantes de otros partidos. En todo caso, el hecho de incluir esto en una u otra dimensión no cambia las conclusiones del análisis. 


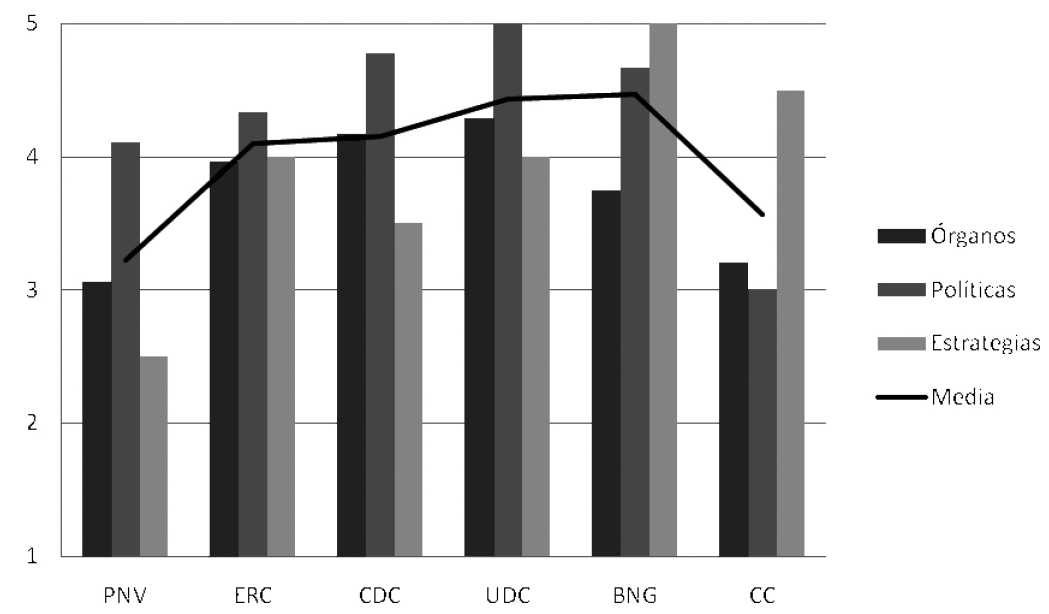

Figura 1. Nivel de integración vertical entre tres dimensiones distintas y media para cinco PANE. Los datos corresponden a los congresos realizados en los siguientes años: PNV, 2004; ERC, 2001; CDC, 2004; UDC, 2004; BNG, 2003; CC, 2005.

La figura 1 muestra los niveles de integración vertical que cinco PANE - Convergència Democràtica de Catalunya (CDC), Unió Democràtica de Catalunya (UDC), Euzko Alderdi Jeltzalea-Partido Nacionalista Vasco (PNV), Bloque Nacionalista Galego (BNG) y Coalición Canaria (CC) — mostraban en 2004 de acuerdo con nuestra clasificación, tanto de media como para cada una de las tres dimensiones analizadas. Como puede observarse, existe amplia variación entre dichos partidos.

De entre todos los PANE señalados anteriormente, el PNV es, seguido de $\mathrm{CC}$, aquel en que más autonomía tienen de media las subunidades territoriales. Esto debería hacer de ellos, según la hipótesis anteriormente apuntada, partidos más proclives a la territorialización interna del faccionalismo. Los bajos niveles de integración vertical del PNV en comparación con el resto de partidos del sistema político español han sido una constante. Si a esto unimos el hecho de que dicho partido presentó unos altos niveles de faccionalismo hasta la década de los noventa y que, además, dicha situación se saldó con la salida de la facción minoritaria, esto lo convierte en un caso apropiado para poner a prueba las hipótesis anteriormente desarrolladas. Digamos que el sistema de partidos nos ha provisto de un caso que resulta, a efectos de este estudio, todo un experimento natural.

\section{La organización del PNV desde 1977 hasta 1987}

En este apartado, analizaremos la regulación estatutaria del PNV en las tres dimensiones anteriormente mencionadas desde 1977 hasta 1987. 
En relación con el grado de integración vertical, los preceptos que regulan el funcionamiento del PNV no cambian durante este periodo, en relación con lo regulado en los primeros estatutos adoptados tras la dictadura (EAJ-PNV, 1977). Estamos hablando de un partido que ya contaba con ochenta y dos años de historia en ese momento y que había tenido un papel relevante durante la Segunda República. Así, los estatutos puestos en marcha tras la transición eran prácticamente un fiel reflejo de la forma confederal de funcionamiento que el partido tenía en el anterior periodo democrático.

Existen distintos factores que explican el bajo nivel de integración vertical de la organización del PNV en su trayectoria prefranquista. En primer lugar, factores ideológicos: la abolición foral y el fuerismo político al que ésta dio lugar fueron condicionantes de los primeros pasos del partido, tanto en la vertiente doctrinal como en la organizativa. Por su raíz tradicionalista y su conceptualización esencialista de la nación, el primer PNV otorgó a la foralidad una posición central en su doctrina y ésta se expresaba en términos provinciales. Por ello, desde el comienzo, los modelos de organización territorial del PNV otorgaban un alto grado de autonomía a los denominados territorios históricos frente a la organización central. Con posterioridad, los contextos provinciales tendieron a divergir más que a converger, tanto en desarrollo económico como sociopolítico, lo que aumentó las presiones centrífugas sobre la organización territorial. Por último, la desigual implantación del PNV — mucho mayor en Vizcaya, y secundariamente en Guipúzcoa, que en Álava y Navarra-, en interacción con sucesivas modalidades de leyes electorales mayoritarias, consolidó la practica de estrategias electorales diferenciadas dependiendo del ámbito provincial: en solitario en Vizcaya; en coalición con otras fuerzas políticas, especialmente el carlismo, en las otras tres provincias. Todos estos factores favorecieron que, bajo la Segunda República, el PNV se consolidará en torno a un modelo de organización que otorgaba una gran autonomía al nivel provincial (De Pablo y Mees, 2005; Pérez-Nievas, 2002). Este modelo organizativo se readoptó, prácticamente sin variaciones, cuando el partido salió de la clandestinidad en 1977.

A continuación, examinaremos, dimensión por dimensión, la organización del PNV para el período comprendido entre 1977 y 1987.

\section{El partido como organización ${ }^{2}$}

Durante este periodo, los estatutos se caracterizan por dotar de gran autonomía a los territorios del partido. La afiliación era administrada por cada uno de los distintos niveles territoriales (art. 5), y lo mismo ocurría con las finanzas. El partido se dividía en cuatro regiones (Álava, Vizcaya, Guipúzcoa y

2. Sobre la organización del PNV en el período 1977-1987, puede consultarse también PérezNievas (2002: 319-326). 
Navarra) ${ }^{3}$, cada una de las cuales gestionaba sus propios recursos y pasaba al centro una cuota determinada de lo recaudado (arts. 52). El centro, por su parte, también tenía capacidad de gestión, y a él correspondían todos los recursos obtenidos en su propio ámbito, es decir, los derivados de la representación del partido en los ámbitos estatal y autonómico (art. 53).

En cuanto a los órganos del partido, podemos distinguir dos tipos: un órgano legislativo (la Asamblea Nacional), cuyas reuniones se celebraban anualmente, y uno ejecutivo (el Euzkadi Buru Batzar, en adelante, EBB). Ambos órganos están conformados íntegramente por representantes elegidos por los territorios en idéntico cupo y al margen del peso electoral o del número de militantes en cada territorio. Cada nivel tiene autonomía para organizarse, elegir los procedimientos de elección de sus propios órganos y marcar su agenda. Y, aunque los estatutos centrales mencionan aspectos relativos a los órganos locales, lo hacen únicamente para indicar los órganos municipales que deben existir en cada región, sin ir más allá (arts. 11-13). La Asamblea Nacional es soberana para decidir los asuntos que le hayan conferido los órganos regionales, además de los que afecten a ámbitos superiores al de dichos territorios. La conforman, con voz y voto, quince representantes de cada una de las regiones, junto con representantes de las llamadas juntas extranacionales, formadas por militantes residentes fuera de los territorios vascos, que no pueden superar el $10 \%$ de la composición del órgano (art. 18). Por su parte, el EBB consta de tres representantes de cada región, que eligen, a su vez, de entre ellos a un portavoz — el presidente del EBB - (arts. 32-36). El EBB tenía, además, la potestad de nombrar varios cargos técnicos dependientes íntegramente del mismo, aunque sin ningún otro poder de carácter político (art. 40). El carácter regional de los representantes, tanto en el EBB como en la Asamblea Nacional, queda marcado en los reglamentos de funcionamiento de los territorios, que los sujetan a mandato imperativo (véase la figura 3 más adelante).

En cuanto a la unidad orgánica del partido, se regula expresamente que las decisiones adoptadas a nivel central deben ser respetadas por todos los territorios. Sin embargo, en cuanto a la resolución de infracciones, la presencia central es mínima: existe una comisión, denominada Tribunal Supremo, que está conformada por un afiliado de cada región y presidida por una persona elegida por el centro (arts. 43-45).

\section{El partido como estructurador de políticas}

En este ámbito, la autonomía de los distintos territorios es prácticamente total. Los estatutos no atribuyen a los órganos centrales ningún papel relevante en

3. Sólo con posterioridad empezará a ser tenida en cuenta la organización del partido en el País Vasco francés, y únicamente tras reformas posteriores a 1987 se alude a ellas estatutariamente y se las incluye como miembros del órgano ejecutivo central, si bien con un número de representantes inferior. La implantación del partido en dicho territorio es muy débil, y solamente ha concurrido a algunas elecciones, no siempre en las mismas circunscripciones, a partir de 1997 con escaso éxito. 
la elaboración de la campaña electoral ni en la adopción de los programas. Tampoco existen mecanismos especiales, aparte de los acuerdos del EBB, para uniformizar el mensaje o las estrategias. Por lo tanto, cada territorio tiene amplio margen de maniobra a este respecto, debiendo únicamente respetar aquellas decisiones tomadas en los órganos centrales (arts. 32 y 70). Hay que tener en cuenta, sin embargo, que éstos están, a su vez, únicamente conformados por representantes regionales que, como tales, rendían cuentas ante los órganos de su correspondiente región. Precisamente estos aspectos centrarán varios de los conflictos más relevantes del periodo.

\section{El partido en las instituciones}

También la autonomía era amplia a este respecto. El nombramiento de los representantes del partido en las instituciones, así como las estrategias de coalición a llevar a cabo, se dejaba íntegramente en manos de los distintos territorios. De hecho, si bien en cuanto al segundo punto, el silencio estatutario podría haber dado lugar a acuerdos entre los territorios en el EBB, la elección de los candidatos se dejaba explícitamente al arbitrio de cada uno de ellos (arts. 56-58). Así, las regiones no sólo elegían a sus candidatos para los órganos de ámbito regional (juntas y diputaciones forales), sino también a cualquier candidato que se presentara por su circunscripción a cualquier tipo de elección, es decir, elecciones autonómicas y generales incluidas. En manos de los órganos centrales quedaba únicamente la elección de cargos cuando ésta se realizaba por circunscripciones mayores que la provincia, lo que, en la práctica, implicaba sólo control directo sobre los candidatos del partido al Parlamento Europeo a partir de 1987, además de cierto poder indirecto para determinar el nombramiento de cargos de confianza del Gobierno Vasco y del propio lehendakari - ya que, al menos formalmente, el aparato del partido tenía potestad para controlar la actividad del grupo parlamentario en las cámaras vasca y navarra, así como en el Congreso de los Diputados.

\section{La organización territorial del faccionalismo en el PNV}

Según lo argumentado con anterioridad, las facciones tenderán a concentrarse territorialmente en partidos poco integrados verticalmente, con lo cual se prioriza la pugna por el control de organizaciones de niveles inferiores al central como forma de contar con recursos suficientes para mantener la lucha interna y asegurarse, al mismo tiempo, cuotas de poder dentro de los órganos centrales.

El caso del PNV es paradigmático en este sentido. La característica esencial de las disputas internas que se suceden desde 1979 hasta 1986 es su territorialización (Pérez-Nievas, 2002). En dicho plazo de tiempo, tienen lugar fundamentalmente dos conflictos entre las facciones del PNV, y los dos se desarrollan claramente de manera territorial. Explicaremos brevemente las dinámicas que se sucedieron en ambos casos. 
El primero de los conflictos tiene lugar a finales de la década de 1970 y se encuentra protagonizado por varias facciones contrarias a las posiciones adoptadas por la facción dominante en el EBB, a la que calificaban de posibilista. $\mathrm{La}$ estrategia de las facciones minoritarias pasaba inexorablemente por penetrar primero en las organizaciones territoriales para, desde allí, pasar al «asalto al partido" (Arzalluz, 2005: 192). El primer paso lo da el llamado sector ormazista, liderado por Antón Ormaza, que, aprovechando que varios miembros de los órganos regionales de Vizcaya han de dejar sus cargos — por imposición estatutaria- para pasar a ser cargos públicos, se hace con el poder de dicho territorio. Esta estrategia es imitada por sectores afines en otros territorios, aunque es el control de la Asamblea Regional de Guipúzcoa por parte de la minoría lo que definitivamente enciende las alarmas en la facción dominante central, puesto que ambos territorios suponían la mitad de los miembros en el EBB. Sin embargo, la limitación de poderes de los órganos centrales a este respecto deriva el conflicto hacia los territorios. El sector ormazista intenta asegurar su poder sobre Vizcaya llevando a cabo varias expulsiones que la facción mayoritaria consigue revocar en los órganos de resolución de conflictos del partido, donde aún conservaban la mayoría. A partir de entonces, éstos últimos deciden concentrar sus esfuerzos en dicha provincia, enviando para ello a uno de sus diputados en el Congreso de los Diputados, Xabier Arzalluz, que, tras varios intentos, consigue recuperar el poder en Vizcaya en la asamblea regional de 1979 (Garaikoetxea, 2002: 75) y posteriormente procede al aislamiento e incluso a la expulsión de los sectores díscolos dentro del territorio ${ }^{4}$, esta vez con el silencio de un EBB cómplice. Así, entre 1980 y 1981, todos los miembros del sector ormazista fueron expulsados del partido (De Pablo y Mees, 2005).

Tras este primer conflicto, en la primera mitad de los ochenta, se fueron perfilando, en el seno del PNV, dos facciones que se hicieron más manifiestas conforme el partido tuvo que hacer frente a distintos tipos de políticas, tanto desde el Gobierno Vasco como desde el Congreso de los Diputados. Uno de dichos sectores estuvo liderado públicamente por Carlos Garaikoetxea, que asumió la presidencia del Gobierno Vasco en 1980 y abogaba por dejar atrás los presupuestos políticos del foralismo, con lo cual aumentaba el poder de las instituciones vascas sobre las provincias, y se mostraba favorable a suscribir acuerdos con partidos de la izquierda, sobre todo del ámbito nacionalista. Por otro lado, Arzalluz, elegido presidente del EBB tras dejar el puesto Garaikoetxea, representaba un segundo sector que defendía los principios más tradicionalistas del PNV, estaba más comprometido con la gobernabilidad del Estado al modo del nacionalismo moderado catalán, apostaba por el foralismo como forma de organización territorial del País Vasco, era favorable a la entrada de España en la OTAN y se decantaba por llegar a entendimientos con la derecha estatal (De Pablo y Mees, 2005; Pérez-Nievas, 2002). 
Como había ocurrido a finales de los setenta, el conflicto entre ambas facciones se reflejó también, durante este periodo, en una ardua batalla por lograr la hegemonía de la organización en los distintos territorios. Al principio de resultar elegido, Garaikoetxea reclamó ser eximido de la disciplina de partido, lo que le permitía saltarse los mandatos del sector de Arzalluz, que controlaba el EBB. Es probable que pensara que esto le daría poder para controlar el partido, o que al menos su visibilidad pública jugaría a su favor. El propio Arzalluz (2005: 213) ha afirmado posteriormente que «[Garaikoetxea] quería todo el poder para él y que el partido se limitara a facilitar el cumplimiento de sus exigencias». Sin embargo, no parece que dicha estrategia resultara exitosa. La coalición dominante central se opuso abiertamente a varias de las decisiones tomadas por el Gobierno Vasco, entre otras, y con gran virulencia, a la tramitación de la Ley de Territorios Históricos, que, en su propuesta original, fortalecía las competencias del Gobierno Vasco frente a las distintas provincias ${ }^{5}$. Tal grado alcanzó el enfrentamiento que dicha ley quedó paralizada. Ante la futilidad que suponía intentar enfrentarse al partido desde los órganos del Gobierno Vasco, el sector de Garaikoetxea decide entonces concentrarse en la lucha de poder dentro de los distintos territorios. Los enfrentamientos con las diputaciones de los territorios controlados por el sector mayoritario central se incrementaron (Garaikoetxea, 2002: 214). Arzalluz se había hecho fuerte en Vizcaya tras el enfrentamiento con Ormaza, su facción era también claramente mayoritaria en Álava y seguía controlando la ejecutiva de Guipúzcoa. Ello le otorgaba una clara mayoría en el EBB; mientras que el sector representado por Garaikoetxea se concentraba en conseguir el control en dos de los territorios donde contaban con más fuerza, Guipúzcoa y Navarra (Pérez-Nievas, 2002: 479-481).

En 1984, la facción dominante aprovechó su mayoría en el EBB para acordar una estrategia de acercamiento al centro y a la derecha estatales, en contra del criterio de los sectores minoritarios, con Garaikoetxea al frente. En un acuerdo con Herrero de Miñón, varios líderes del sector mayoritario se habían comprometido a facilitar un gobierno conservador en Navarra a cambio de que la Coalición Popular de Fraga hiciera lo mismo a favor del PNV en varios ayuntamientos y juntas generales, como también en el Parlamento de Navarra (De Pablo y Mees, 2005; Garaikoetxea, 2002). Para llevarlo a cabo, la coalición dominante forzó la votación de dicha decisión en el EBB, pero la organización navarra — controlada ya por el sector de Garaikoetxea - no asumió la decisión y se negó a permitir con sus votos un gobierno presidido por la Unión del Pueblo Navarro (UPN), con lo cual dejó de facto el gobierno navarro en manos del PSOE. Lo cierto es que los movimientos del sector minoritario en Guipúzcoa amenazaban el control del EBB por parte de la facción mayoritaria ${ }^{6}$, así que dicho órgano respondió a la actitud de los navarros con

5. El País, 2 de enero de 1984.

6. El hecho de que se estaban realizando esfuerzos en ese sentido puede inferirse de las palabras de Garaikoetxea (2002: 223) cuando afirma que «[a]unque en Guipuzkoa los representantes en los órganos nacionales mantenían una postura de total identificación con la 
la disolución forzada de sus órganos regionales y la creación de una comisión gestora afín a la mayoría del EBB, con lo cual se dio la paradoja de que, en un partido cuyo funcionamiento era claramente confederal, se castigaba al territorio navarro por querer ejercer autonomía dentro de su propia comunidad autónoma (Garaikoetxea, 2002).

A partir de ese momento, las luchas internas se incrementan dentro del partido, y el enfrentamiento entre el mismo y el Gobierno Vasco, liderado por Garaikoetxea, llega a tal punto que la Asamblea Nacional llega a retirar la confianza al propio lehendakari, que se ve forzado a dimitir y es sustituido por un parlamentario de confianza del EBB, José Antonio Ardanza ${ }^{7}$.

La territorialización del conflicto sigue siendo clara durante los años siguientes. Dado que cada región era autónoma para regular la composición de sus órganos, sucedía que, tanto en Álava como en Guipúzcoa, las juntas municipales urbanas apoyaban a Garaikoetxea, pero, al tener el mismo peso que las juntas rurales, las ejecutivas de ambos territorios se encontraban totalmente controladas por la facción dominante del partido. A pesar de ello, en 1985, los críticos lograron hacerse finalmente con la ejecutiva guipuzcoana, lo cual forzó la sustitución de todos sus miembros en el EBB y se insistió en que se tramitara la readmisión de los navarros expulsados, lo que habría puesto en jaque a la coalición dominante, que, como es obvio, se negó a llevarlo a cabo (De Pablo y Mees, 2005). Controlada Navarra, Guipúzcoa se encontraba acorralada en los órganos centrales, lo que hacía prever la exclusión de la facción minoritaria en las listas de las elecciones autonómicas de ese mismo año. Ante esta situación, la organización territorial del PNV en Guipúzcoa decide escindirse definitivamente del partido y crear lo que acabará siendo EA.

En todo momento, las facciones situaron el control de los territorios como una prioridad estratégica y concentraron sus esfuerzos sobre los órganos de las subunidades regionales. A ello hay que unir la instrumentación, por parte de

Tabla 2. Afiliados al PNV $(1985,1996)$ y a EA (1986) por territorios históricos o provincias

\begin{tabular}{lrrrrr}
\hline & \multicolumn{2}{c}{ PNV } & & EA & \\
\cline { 2 - 3 } & \multicolumn{1}{c}{$\mathbf{1 9 8 5}$} & $\mathbf{1 9 9 6}$ & & $\mathbf{1 9 9 6}$ & Ratio EA/PNV \\
\hline Vizcaya & 23.500 & 24.152 & & 6.883 & 0,28 \\
Guipúzcoa & 8.000 & 4.036 & & 5.496 & 1,36 \\
Álava & 1.500 & 2.132 & & 1.312 & 0,62 \\
Navarra & 1.000 & 167 & & 1.120 & 6,71 \\
Total & 34.000 & 30.487 & & 14.811 & 0,49 \\
\hline
\end{tabular}

Fuentes: para 1985, Letamendía (1994: 52); para 1996, Acha y Pérez-Nievas (1998: 97).

dirección del partido, como luego se demostraría, la organización guipuzcoana se decantaba por abrumadora mayoría en su contra».

7. El País, 23 de enero de 1985. 
ambas facciones, de la organización del PNV. Mientras que, por un lado, quienes tenían la mayoría en el aparato del partido, es decir, el sector liderado por Arzalluz, defendía un modelo más centralizado que asumiera las decisiones tomadas en el EBB, el sector afín a Garaikoetxea defendía una mayor autonomía interna de las organizaciones regionales. Unos y otros acabarían defendiendo, por tanto, modelos antagónicos para la organización del partido, por un lado, y para las instituciones autonómicas, por otro (Pérez-Nievas 2002).

Lo óptimo para ilustrar el grado en que cada una de las facciones había conseguido asentarse organizativamente en cada territorio sería contar con los porcentajes de afiliación que pasaron directamente a engrosar las filas de EA tras la ruptura. Aunque no contamos con esos datos para 1986, en 1996, las tendencias aún parecen claras (véase la tabla 2). Como puede observarse, la implantación del partido escindido, EA, se concentra sobre todo en los territorios de Navarra y Guipúzcoa. A pesar de contar en total con la mitad de afiliados que el PNV, en dichos territorios, EA tenía en 1996, respectivamente, 6,71 y 1,36 veces los afiliados del primero. En los otros dos territorios, controlados por el sector mayoritario del EBB, es el PNV quien obtiene ventaja sobre EA. Esto da cierta evidencia del grado de concentración territorial que habían alcanzado las distintas facciones tras años de luchas internas. Por tanto, parece que el caso del PNV nos permite apoyar la hipótesis de la territorialización del conflicto faccional. Ahora bien, para contrastar la segunda hipótesis planteada, es necesario atender a la evolución organizativa del partido una vez producida la ruptura.

\section{Las consecuencias de la ruptura sobre el grado de integración vertical del partido}

Si la hipótesis de partida estaba en lo cierto, tras una ruptura como la sufrida por el PNV a finales de la década de 1990, el partido debería experimentar cambios organizativos dirigidos a aumentar el control del centro sobre las decisiones. Efectivamente, esto es lo que se infiere de la evolución del PNV posterior a la salida de EA. Los estatutos, que durante diez años permanecieron estáticos en relación con la integración vertical del partido, cambiaron en la Asamblea Nacional Extraordinaria celebrada el 31 de octubre de 1987, cuando se reforzó el poder del centro y se matizó la estructura confederal previa. Las posteriores reformas estatutarias de 1992 y 1995 reforzaron aún más los esfuerzos de centralización del partido que introdujeron los estatutos de 1987. La figura 2 representa, de forma dinámica, los cambios habidos en el PNV en relación con cada una de las dimensiones analizadas con anterioridad desde 1977 hasta 2003. Tal y como se muestra, el año 1987 aparece definitivamente como punto de inflexión en el desarrollo organizativo del PNV en relación con todas y cada una de las tres dimensiones analizadas — si bien en la dimensión «estrategias» los cambios parecen ser más suaves- . De hecho, los niveles de integración vertical del PNV, a pesar de ciertas variaciones, no vuelven ya a los niveles anteriores a la escisión. 


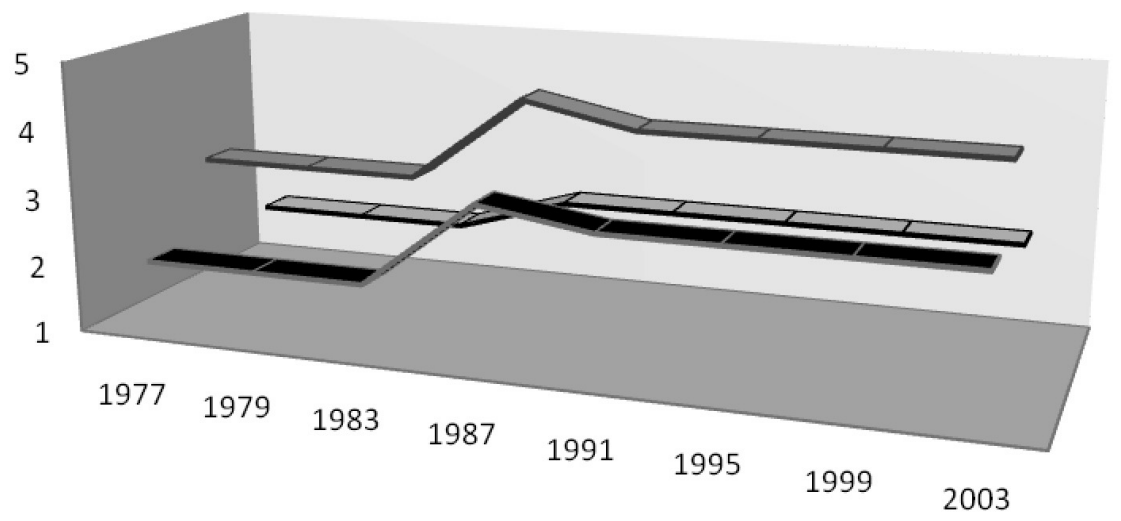

- órganos $\square$ políticas $\square$ estrategias

Figura 2. Evolución organizativa del PNV de 1977 a 2003. Los años en el eje de abscisas se corresponden con las legislaturas del Parlamento vasco.

A continuación, procederemos a desgranar, dimensión por dimensión, el contenido de los cambios encontrados en los primeros estatutos adoptados tras la ruptura (EAJ-PNV, 1987), comparándolos con la situación previa.

\section{El partido como organización tras la escisión de $E A^{8}$}

Las modificaciones llevadas a cabo en 1987 en esta dimensión ejemplifican claramente el aumento del poder del centro. Existen cambios destacables, como es el hecho de que por primera vez se establezca que sea un órgano central, la Asamblea Nacional, quien fije las aportaciones mínimas, procedentes tanto de las cuotas de afiliación como de otros ingresos, que cada organización territorial debe realizar a los órganos centrales (art. 100). Pero quizá el aspecto más relevante en este ámbito es la total reorganización que, desde este momento, se hace de los órganos del partido (véase la figura 2). En primer lugar, se acaba con el sistema confederal que concedía los mismos representantes a cada una de las organizaciones territoriales. Tras la escisión de EA, se adopta un sistema consistente en elegir, para la Asamblea Nacional, un número fijo de delegados de cada territorio más otro número proporcional al número de afiliados de cada uno (art. 38). La Asamblea Nacional sigue convocándose de manera ordinaria con una periodicidad anual, pero, además de ella, se introduce un

8. Sobre la organización del PNV tras la escisión de 1987, puede consultarse también PérezNievas (2002: 555-561). 
nuevo órgano llamado Asamblea General (arts. 46-48), con las características de un congreso, cuyas reuniones se celebran cada cuatro años. Es de destacar que, aparte de quienes componen la Asamblea Nacional saliente y de varios miembros más - representación de cargos públicos y de Euzko Gaztedi, las juventudes del PNV-, los representantes de la Asamblea General son elegidos en función de un sistema proporcional desde cada organización municipal (art. 47), lo que disminuye el poder que las organizaciones regionales tenían hasta entonces en los órganos centrales del partido. Por otro lado, se especifica que, para la toma de decisiones en ciertos asuntos, la Asamblea General deberá consultar directamente a las bases a través de las asambleas municipales (art. 49). De nuevo, se trata de un mecanismo que une directamente al órgano central con los órganos de base, sin necesidad de pasar por la mediación de los órganos regionales (véase la figura 3 ).

También en la composición del EBB prácticamente se pone fin al sistema confederal. Si bien con anterioridad este órgano estaba formado por tres representantes en cada región, que respondían en la práctica a sus órganos territoriales, a partir de 1987 se completa el órgano con ocho miembros que, a pesar de ser propuestos por las asambleas regionales, son directamente elegidos por la Asamblea Nacional. Además de ellos, componen el órgano los presidentes de los consejos regionales, junto con el presidente del EBB (art. 58).

Cambios de similar entidad se producen también en el Tribunal Supremo, cuya composición pasa a ser de cinco personas y son elegidas en su totalidad por la Asamblea Nacional, con lo cual se sustrae esta potestad a las regiones (art. 74). La figura 3 muestra gráficamente el cambio en el organigrama del partido a través de las sucesivas reformas de 1987, 1992 y 1995.

\section{El partido como estructurador de politicas}

En relación con la oferta política, los nuevos cambios se mueven en la misma dirección apuntada anteriormente. Por un lado, el EBB se arroga ahora no sólo la aprobación de los programas electorales, sino también la dirección de las campañas (art. 59 c y d), en contraste con la anterior autonomía que existía al respecto. Además de ello, se regula claramente que todos los órganos deben respetar las decisiones tomadas a nivel central, con lo cual se deja en manos del EBB no sólo la política de comunicación, como hasta ahora, sino también la imagen del partido hacia el exterior, y se delimita el poder de los órganos regionales a los asuntos correspondientes al ámbito exclusivo de su territorio. Aún así, deben atenerse a los acuerdos de carácter general tomados por los órganos centrales (art. 102).

Por último, se adopta por primera vez un sistema de coordinación de políticas, de nuevo controlado por el centro. Se trata de reuniones del EBB con cargos públicos de ámbito tanto autonómico como superior, reguladas por un reglamento interno establecido por el centro y a efectos de «facilitar una actuación política coordinada y eficaz» (art. 63). 

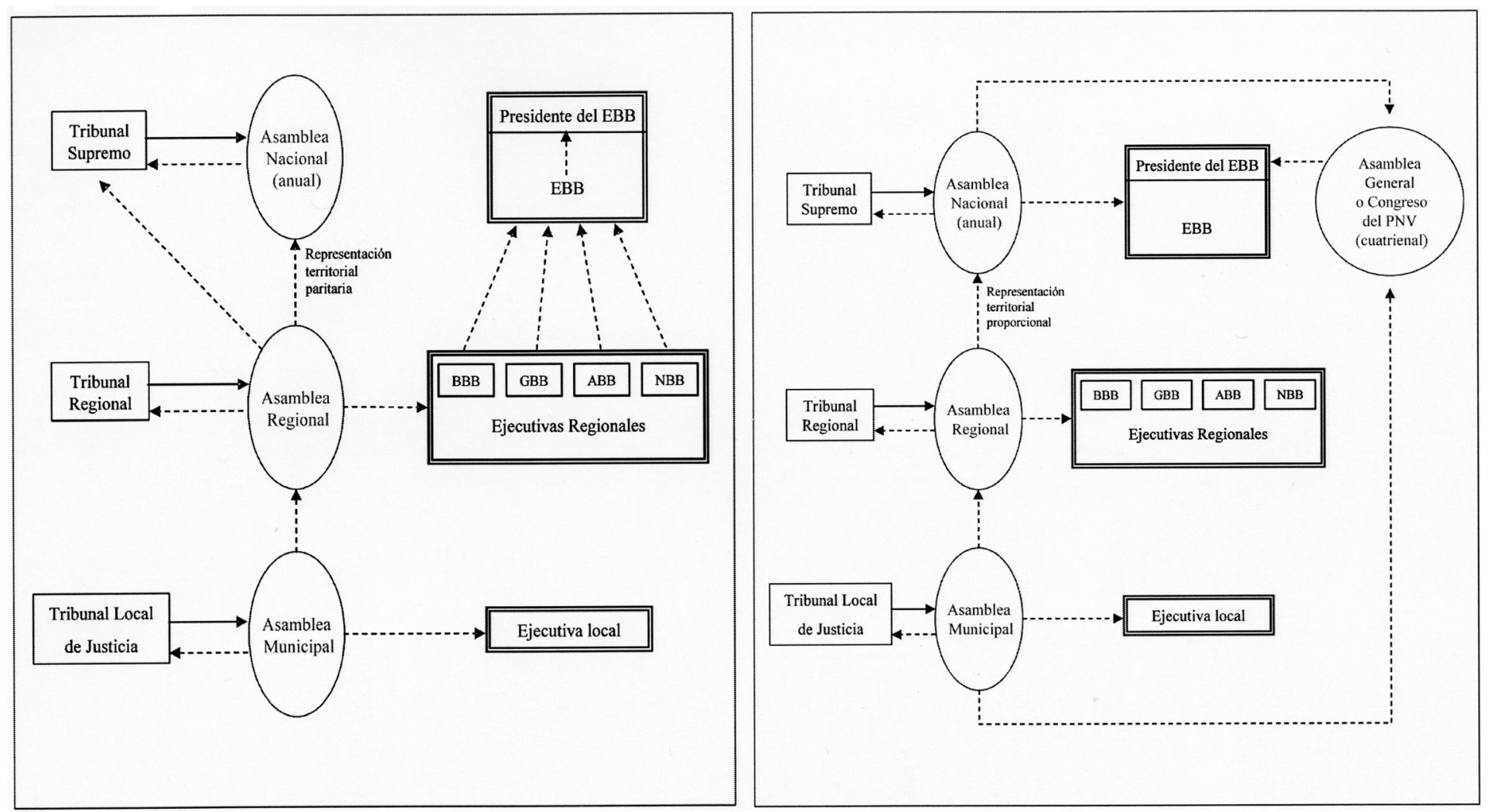

Figura 3. La organización del PNV en el período 1977-1987 (izquierda) y después de 1987 (derecha). Fuente: elaboración propia a partir de los Estatutos de Organización del PNV de 1977, 1987, 1992 y 1995. 


\section{El partido en las instituciones}

También en esta dimensión es de destacar el aumento de las competencias del centro en detrimento de los distintos territorios. A partir de 1987, no sólo se arroga el EBB la potestad de aprobar los acuerdos de gobierno e incluso los pactos parlamentarios de carácter estable en su propio ámbito, sino que también deberá decidir sobre ellos cuando afecten a más de una institución regional, o incluso a más de una institución municipal, en caso de que éstas pertenezcan a regiones diferentes (art. 59 e). Algo similar ocurrirá también con el proceso de selección de candidatos a las distintas instituciones. Si bien con anterioridad cada territorio decidía de forma autónoma las candidaturas de su circunscripción a cualquier tipo de elección, a partir de ahora queda en manos de los órganos centrales — concretamente, de la Asamblea Nacional a propuesta del EBB - la elección de todas las candidaturas a cargos autonómicos o superiores, independientemente de la circunscripción por la que se presenten y sean de elección popular o no, así como la orientación y el control de los mismos (art. 86).

\section{Conclusiones}

El caso del PNV es un claro ejemplo de las dinámicas desarrolladas por las facciones en partidos políticos con un escaso nivel de integración vertical. Tal y como se ha planteado, un mayor nivel de autonomía interna provee de grandes incentivos para que los conflictos faccionales se territorialicen. Y, al menos en el caso del PNV, esto es lo que parece haber sucedido durante el periodo estudiado. Es más, las consecuencias de la escisión de la facción minoritaria del partido apoya, de forma indirecta, los mecanismos aquí mantenidos para explicar la relación entre integración vertical y el faccionalismo dentro de los partidos.

En todo caso, el interés de este estudio se concentra básicamente en dos pilares distintos: el primero está vinculado con las hipótesis planteadas y la evidencia empírica encontrada, mientras que el segundo tiene que ver con la aplicación de nuestras hipótesis a un partido de ámbito estatal.

Efectivamente, los mecanismos y las hipótesis aquí planteados pueden contrastarse en otros casos. Si tenemos en cuenta el caso de Izquierda Unida, que comparte ciertos rasgos organizativos con el PNV, lo cierto es que las dinámicas con que el faccionalismo se ha desarrollado en ambos partidos son similares: en los dos destaca la territorialización del mismo y una instrumentalización de cuestiones organizativas relacionadas con el grado de integración vertical por parte del conjunto de facciones. Por otro lado, el alto grado de faccionalismo limita las reformas estructurales del partido en ambos casos, algo que provoca que la facción dominante desarrolle de facto una suerte de integración vertical asimétrica. Mientras que, por un lado, intenta hacer uso de su posición mayoritaria en los órganos centrales para intervenir sobre los territorios díscolos, por el otro se respeta, e incluso se impulsa, el desarrollo de la autonomía de 
sus territorios afines ${ }^{9}$. Nuestro trabajo ha tratado de profundizar en el estudio de las dinámicas aquí expuestas, pero es necesario examinar más evidencia empírica y analizar el funcionamiento del faccionalismo en partidos con distintos niveles de integración vertical.

Por otro lado, debe destacarse la utilidad de estudiar un partido de ámbito no estatal. En primer lugar, por el interés intrínseco de indagar sobre sus formas de funcionamiento y explorar sobre las diferencias y las similitudes con los partidos de ámbito estatal. Y, en segundo lugar, porque este tipo de partidos se encuentran en gran medida infraestudiados. Independientemente del ámbito en que actúen, los partidos no dejan de ser un mismo tipo de actor político, y el gran número de PANE existente en sistemas políticos como el español proporciona una gran cantidad de información que está siendo desaprovechada y que nos permitiría realizar investigaciones comparadas con un número de casos nada desdeñable para alguien dedicado al estudio de los partidos, con la ventaja adicional de mantener constante gran parte del contexto institucional bajo el cual actúan nuestros objetos de estudio.

\section{Referencias bibliográficas}

ACHA, B.; PÉreZ-Nievas, S. (1998). «Moderate nationalist parties in the Basque Country: Partido Nacionalista Vasco and Eusko Alkartasuna». En: DE WINTER, L.; H. TÜRSAN (eds.). Regionalist parties in Western Europe. Londres: Routledge. ArZAlluZ, X. (2005). Asi fue. Madrid: Foca.

AsTUDILlO, J. (en prensa). «La distribución territorial del poder en los partidos políticos: el caso del Partido Popular español». En: F. PALlarés (ed.). Competición politica multinivel en el Estado de las Autonomías: Ciudadanos, partidos y elecciones. Valencia: Tirant Lo Blanch.

BarberÀ, Ò.; Sallas, M.; Terrades, M. (2007). Descentralización política y partidos implantados en una sola región (PANE): marco de análisis y evidencias empiricas preliminares. Ponencia presentada en el VIII Congreso de la Asociación Española de Ciencia Política y de la Administración. Valencia, 18-20 de septiembre.

BARNEA, S.; RAHAT, G. (2007). «Reforming candidate selection methods: A ThreeLevel Approach». Party Politics, 13(3), 375-394.

BETANZO, A. (2006). «Los efectos de la transformación territorial del Estado español sobre el PSOE y el PP». Revista d'Estudis Autonòmics i Federals, 3, 223-258.

CARTY, R. K. (1988). «Three Canadian party systems: An interpretation of the development of national politics». En: G. PERLIN (ed.). Party democracy in Canada. The politics of national party conventions. Scarborough: Prentice Hall.

DESCHOUWER, K. (2003). «Political parties in multi-layered systems». European Urban and Regional Studies, 10(3), 213-226.

DYCK, R. (1991). "Links between federal and provincial parties and party systems». En: H. BAKVIS (ed.). Representation, integration and political parties in Canada. Toronto: Dundum Press.

9. Para el caso de Izquierda Unida, ver Verge (2007) y Resolución de la Presidencia Ejecutiva Federal de IU, 18 de diciembre de 2007. 
EAJ-PNV (1977). Ponencia de organización. Texto aprobado en la Asamblea Nacional de Iruña el día 26 de marzo de 1977.

EAJ-PNV (1987). Estatutos Nacionales.

EAJ-PNV (1992). Estatutos Nacionales.

EAJ-PNV (1995). Estatutos Nacionales.

GaraikoetXeA, C. (2002). Euskadi: La transición inacabada. Barcelona: Planeta.

GÓMEZ, R. (2007). La organización de los partidos en sistemas multinivel. Evidencia comparada para el caso español. Ponencia presentada en el VII Congreso de la Asociación Española de Ciencia Política. Valencia, 18-20 de septiembre.

Hopkin, J. (2003). «Political decentralization, electoral change and party organisational adaptation: A framework for analysis». European Urban and Regional Studies, 10(3), 227-237.

Hopkin, J.; VAN BIEZEN, I. (2006). «Party organization in multilevel contexts». En: D. Hough; C. JefFery (eds.). Devolution and electoral politics. Manchester: Manchester University Press.

Hough, D.; JefFery, C. (eds.) (2006). Devolution and electoral politics. Manchester: Manchester University Press.

LAFFin, M.; SHAW, E.; TAYLOR, G. (2007). «The new sub-national politics of the British Labour Party». Party Politics, 13(1), 88-108.

LETAMENDÍA, F. (1994). Historia del nacionalismo vasco y de ETA. ETA en la transición (1976-1982). San Sebastián: R \& B Ediciones.

MéndeZ, M. (2004). Federalismo y partidos politicos: los casos de España y Canadá, vol. 232. Barcelona: Institut de Ciències Polítiques y Socials.

MÉNDEZ, M.; ORTE, A. (en prensa). «La organización de partidos en sistemas multinivel: el caso del PSOE». En: F. PALLARÉS (ed.). Competición política multinivel en el Estado de las Autonomías: Ciudadanos, partidos y elecciones. Valencia: Tirant Lo Blanch.

Pablo, S. DE; MeEs, L. (2005). El péndulo patriótico. Historia del Partido Nacionalista Vasco (1895-2005). Barcelona: Crítica.

PÉreZ-Nievas, S. (2002). Modelo de partido y cambio político. El Partido Nacionalista Vasco en el proceso de transición y consolidación democrática en el País Vasco. Madrid: Instituto Juan March de Estudios e Investigaciones.

PÉREZ-NievaS, S.; RAmiro-FernÁndeZ, L. (en prensa). «El impacto de los procesos de descentralización territorial en la organización de los partidos políticos: el caso del Izquierda Unida». En: F. PALlarés (ed.). Competición politica multinivel en el Estado de las Autonomias: Ciudadanos, partidos y elecciones. Valencia: Tirant lo Blanch.

SMiLeY, D. V. (1987). The federal condition in Canada. Toronto: Mc Graw-Hill Ryerson. ŞTEFURIUC, I.; VERGE, T. (2008). «Small and divided parties in multi-level settings: Opportunities for government participation, the case of Izquierda Unida in Spain». South European Society and Politics, 13 (2): 155-173.

THORLAKSON, L. (2001). Federalism and party organisational adaptation: a cross-national comparison. Trabajo presentado en el ECPR Joint Sessions of Workshops. Grenoble (Francia).

Verge, T. (2007). «Is United Left becoming a party of government?». Presentado a Political Science Association (PSA) Conference, University of Bath, 11-13 de abril de 2007. 